preprint - UTF 377

\title{
Quantum Fields in Hyperbolic Space-Times with Finite Spatial Volume
}

\author{
Andrei A. Bytsenko \\ State Technical University, St. Petersburg 195251, Russia \\ Guido Cognola 草 and Sergio Zerbini 过 \\ Dipartimento di Fisica, Università di Trento \\ and Istituto Nazionale di Fisica Nucleare, \\ Gruppo Collegato di Trento, Italia
}

May 1996

Abstract: The one-loop effective action for a massive self-interacting scalar field is investigated in 4-dimensional ultrastatic space-time $R \times H^{3} / \Gamma, H^{3} / \Gamma$ being a non-compact hyperbolic manifold with finite volume. Making use of the Selberg trace formula, the $\zeta$-function related to the small disturbance operator is constructed. For an arbitrary gravitational coupling, it is found that $\zeta(s)$ has a simple pole at $s=0$. The one-loop effective action is analysed by means of proper-time regularisations and the one-loop divergences are explicitly found. It is pointed out that, in this special case, also $\zeta$-function regularisation requires a divergent counterterm, which however is not necessary in the free massless conformal invariant coupling case. Finite temperature effects are studied and the high-temperature expansion is presented. A possible application to the problem of the divergences of the entanglement entropy for a free massless scalar field in a Rindler-like space-time is briefly discussed.

PACS numbers: $\quad 04.62 .+v, 11.10 . W \mathrm{x}$

\section{Introduction}

In the last decades there has been a lot of investigations about the properties of quantum fields in curved space-times [1,2]. There has also been some interest in studying free scalar fields in static topologically nontrivial space-times. We would like to remind that a lot of investigations have been concerned with space-times with the toroidal topology (see for example Refs. [3, 国] and references therein) or orbifold factors of spheres [5, 6]. The case of compact hyperbolic manifolds has also been considered (see for example Refs. 近, 面). In this case one is dealing with an ultrastatic space-time $R \times H^{3} / \Gamma, H^{3} / \Gamma$ being a compact hyperbolic manifold, $H^{3}$ the Lobachevsky space and $\Gamma$ a discrete group of isometries acting on $H^{3}$ and containing loxodromic, hyperbolic and elliptic elements (see Refs. [8, 9, 10, 11, 7]).

*email: abyts@spin.hop.stu.neva.ru

${ }^{\dagger}$ e-mail: cognola@science.unitn.it

${ }^{\ddagger} \mathrm{e}-\mathrm{mail}$ : zerbini@science.unitn.it 
In this paper we extend the analysis considering a self-interacting scalar field in an ultrastatic space-times with non-compact, but with finite volume hyperbolic spatial section. Such spaces, for a fixed value of the cosmological time, may be of significant interest in cosmology [12], since it has been recently claimed that hyperbolic compact space-times have some problem with regard to inflationary scenario [13]. In our example, the discrete group of isometry can be chosen in the form $\Gamma=S L(2, \mathbb{Z}+i \mathbb{Z}) /\{ \pm I d\}$. It is generated by parabolic mappings (Id is an isolated identity element of $\Gamma$ ) and is associated with a non-compact manifold having an invariant fundamental domain of finite volume. In fact there exists a homeomorphism between $H^{3} / \Gamma$ and the manifold without boundary $\left(S^{1} \times U_{c}\right) / \partial M, U_{c}$ being a punctured cylinder and the boundary $\partial M$ being homeomorphic to the torus $S^{1} \times S^{1}$.

We shall work in the one-loop approximation. Making use of the Selberg trace formula, we will investigate the asymptotic expansion of the heat-kernel trace $\operatorname{Tr} \exp \left(-t L_{4}\right), L_{4}$ being the small disturbance (Laplace-like) operator. We shall find that the presence of parabolic elements in $\Gamma$ leads to the appearance of logarithmic factor in the small $t$ asymptotic expansion. For non-compact Riemannian surfaces of finite area, this fact has been observed in Refs. [14, 15]. However in this case the meromorphic continuation of the $\zeta$-function has been shown to be regular at $s=0$, thus the determinant of the Laplacian has been evaluated by means of the standard $\zeta$-function regularisation [16, 17]. On the contrary, in the $(1+3)$-dimensional case, we shall show that $\zeta\left(s \mid L_{4}\right)$ is a meromorphic function having a simple pole at $s=0$. In a different context, i.e. the computation of functionals determinant of Laplacian on the generalised cone, the appearance of a non standard logarithmic term has been recently pointed out in Ref. [18] and this fact was first noted by Cheeger [19] and others authors [20, 21]. However, in Ref. [18] the things have been arranged in order to avoid the logarithmic term, by dealing with a conformally coupled free massless field. We will show that also in our case, the same choice leads to a regular $\zeta$-function at $s=0$.

In order to give a meaning to the determinant of the small disturbance operator, we shall make use of the proper-time Schwinger regularisation, which includes the $\zeta$-function one. Within this approach, the one-loop ultraviolet divergences will be explicitly calculated. It will be found that also the regularisation requires a counterterm, which, however, is absent for a conformally coupled free massless scalar field. Besides the usual ones we find new divergences, which, however, may be absorbed making use of the one-loop renormalisation procedure.

The finite temperature effects as well as the vacuum energy will be studied in some detail for a self-interacting scalar field. In absence of parabolic element, these effects have been investigated in Ref. [22]. We find that, in the high-temperature expansion, the parabolic elements induce new terms, which may play a role in the cosmological applications.

The contents of the paper are the following. In Sec. 2 the trace of heat kernel for a Laplacetype operator is computed by means of the Selberg trace formula. The meromorphic structure of the related $\zeta$-function is determined. In Sec. 3 the regularisation of the one-loop effective action is discussed in the framework of the Schwinger regularisations. In Sec. 4 the finite temperature effects are investigated. Finally we end with some conclusions in Sec. 5. In the Appendices, a summary of the Selberg trace formula for a non-compact hyperbolic manifold with finite invariant fundamental domain is presented.

\section{The heat kernel and the $\zeta$-function}

Here we concentrate our attention on a self-interacting scalar field in an ultrastatic space-time $\mathcal{M}$ in which the spatial section is a manifold with constant curvature. For the sake of generality, we shall derive a general expression for the one-loop effective potential. Then we shall discuss in detail the case in which the spatial section is $H^{3} / \Gamma$, the discrete group of isometries $\Gamma$ containing parabolic elements only. When $\Gamma$ contains elliptic elements, conical-like singularities appear in 
the manifold and in this case the renormalisation becomes quite delicate. For a discussion of similar situations see for example Ref. [23].

We start with the classical Euclidean action for the scalar field in $\mathbb{R} \times H^{3} / \Gamma$

$$
S[\phi, g]=\int_{\mathcal{M}}\left[-\frac{1}{2} \phi \Delta \phi+V_{c}(\phi)\right] \sqrt{g} d^{4} x,
$$

where the classical potential reads

$$
V_{c}(\phi)=\frac{\lambda \phi^{4}}{24}+\frac{m^{2} \phi^{2}}{2}+\frac{\xi R \phi^{2}}{2}
$$

$\Delta$ being the Laplace-Beltrami operator, $R$ the scalar curvature, $m$ the mass, $\lambda$ and $\xi$ arbitrary constants. The small disturbance operator associated with the action (2.1) is given by

$$
L_{4}=-\Delta_{4}+V_{c}^{\prime \prime}\left(\phi_{c}\right)=-\Delta_{4}+\alpha^{2},
$$

where we assume $\alpha^{2}=m^{2}+\xi R+\lambda \phi_{c}^{2} / 2$ to be a constant and the prime stands for the derivative with respect to the classical field $\phi_{c}$. Since we are dealing with an ultrastatic space-time, we have

$$
L_{4}=-\partial_{\tau}^{2}-\Delta_{3}+\alpha^{2}=-\partial_{\tau}^{2}+L_{3},
$$

$\Delta_{3}$ being the Laplace-Beltrami operator acting in $H^{3} / \Gamma$.

The one-loop effective potential is defined by

$$
V_{e f f}\left(\phi_{c}\right)=V\left(\phi_{c}\right)+\frac{1}{2 \ell V(F)} \ln \operatorname{det} \frac{L_{4}}{\mu^{2}},
$$

where $\mu$ is a dimensional renormalisation parameter, $\ell$ the (infinite) interval of imaginary time and $V(F)$ the volume of the fundamental domain of $H^{3} / \Gamma$ which is defined in the Appendix A.

As discussed in the Introduction, the determinant of an elliptic differential operator requires a regularisation. One of the most used regularisation is the $\zeta$-function regularisation [16, 24, 17]. By means of it one has

$$
\ln \operatorname{det} \frac{L_{4}}{\mu^{2}}=-\zeta^{\prime}\left(0 \mid \frac{L_{4}}{\mu^{2}}\right)
$$

where the $\zeta^{\prime}$ is the derivative with respect to $s$ of the $\zeta$-function. In the standard cases, the $\zeta$-function at $s=0$ is well defined and so by means of the latter formula one gets a finite result.

It is well known that the meromorphic structure of the analytically continued $\zeta$-function, as well as the ultraviolet divergences of the one-loop effective action, can be related to the asymptotic properties of the heat-kernel trace, which in our case reads

$$
\operatorname{Tr} \exp \left(-t L_{4}\right)=\frac{\ell}{\sqrt{4 \pi t}} \operatorname{Tr} \exp \left(-t L_{3}\right) .
$$

For the rank-1 symmetric space $H^{3} / \Gamma$ (the group $\Gamma$ contains the identity, hyperbolic and parabolic elements) the trace of the operator exp - $\left(t L_{3}\right)$ may be computed by using Eq. (B.15) with the choice $h(r)=\exp \left[-t\left(r^{2}+\delta^{2}\right)\right]$ and $\delta^{2}=|\kappa|+\alpha^{2}$ (here $\kappa=R / 6$ is the (negative) constant curvature of $\left.H^{3}\right)$. We have

$$
g(u)=\frac{e^{-t \delta^{2}} e^{-u^{2} / 4 t}}{\sqrt{4 \pi t}}, \quad g(0)=\frac{e^{-t \delta^{2}}}{\sqrt{4 \pi t}}, \quad h(0)=e^{-t \delta^{2}} .
$$

As a result, directly using Eq. (B.16)

$$
\begin{gathered}
\operatorname{Tr} e^{-t L_{3}}=e^{-t \delta^{2}}\left[\tilde{P}_{1} \ln t t^{-1 / 2}+\tilde{A}_{0} t^{-3 / 2}+\tilde{A}_{1} t^{-1 / 2}-\frac{t}{\pi i} \int_{-\infty}^{\infty} e^{-t r^{2}} f\left(\frac{i r}{2}\right) r d r\right] \\
+\frac{e^{-t \delta^{2}}}{\sqrt{4 \pi t}} \sum_{\gamma} \sum_{k=1}^{\infty} \frac{\chi(\gamma)}{S_{3}(\gamma, k)} \exp \left(-\frac{k^{2} l_{\gamma}^{2}}{4 t}\right)
\end{gathered}
$$


where

$$
\tilde{P}_{1}=\frac{\sqrt{|\kappa|}}{8 \sqrt{\pi}}, \quad \tilde{A}_{0}=\frac{V(F)}{(4 \pi)^{3 / 2}}, \quad \tilde{A}_{1}=\frac{\sqrt{|\kappa|}}{\sqrt{4 \pi}}\left(C+\ln 2+\frac{\gamma}{4}\right),
$$

$C$ being a computable constant (see Appendix B) and $\gamma$ being the Euler constant. The asymptotic behaviour of the last integral for $t \rightarrow 0$ can be evaluated by using Eqs. (2.7) and (B.17) and noting that the last contribution, namely the one associated with hyperbolic elements, is exponentially small (for more details we refer the reader to Ref. [7]). Besides the usual terms one has for the heat kernel in 3-dimensions, one gets terms with logarithmic factors due to the presence of parabolic elements in $\Gamma$. These terms are absent for co-compact group $\Gamma$ (compact hyperbolic manifolds). The asymptotic for the heat kernel reads

$$
\begin{aligned}
\operatorname{Tr} e^{-t L_{3}} & \simeq e^{-t \delta^{2}}\left[\tilde{P}_{1} \ln t t^{-1 / 2}+\tilde{A}_{0} t^{-3 / 2}+\tilde{A}_{1} t^{-1 / 2}+\sum_{n=2}^{\infty} \tilde{A}_{n} t^{n-\frac{3}{2}}\right] \\
& \simeq \sum_{r=0}^{\infty}\left(A_{r}+P_{r} \ln t\right) t^{r-\frac{3}{2}}
\end{aligned}
$$

where $\tilde{A}_{2}=\frac{1}{6 \sqrt{|\kappa| \pi}}$ and all the other coefficients are in priciple computable. We have put

$$
A_{r}=\sum_{n=0}^{r} \frac{(-1)^{n} \tilde{A}_{r-n} \delta^{2 n}}{n !}, \quad P_{0}=0, \quad P_{r}=\frac{(-1)^{r-1} \sqrt{|\kappa|} \delta^{2(r-1)}}{8 \sqrt{\pi}(r-1) !} .
$$

One has a similar short $t$-expansion for the heat-kernel trace related to the operator $L_{4}$. From Eqs. (2.5) and (2.9) one has

$$
\operatorname{Tr} e^{-t L_{4}} \simeq \frac{\ell}{\sqrt{4 \pi}} \sum_{r=0}^{\infty}\left(A_{r}+P_{r} \ln t\right) t^{r-2}
$$

Let us analyse the consequences of the presence of logarithmic terms in the expansion Eq. (2.11). As usual, we may introduce the $\zeta$-function associated with the elliptic operator $L_{4}$ by means of the Mellin transform

$$
\zeta\left(s \mid \frac{L_{4}}{\mu^{2}}\right)=\frac{1}{\Gamma(s)} \int_{0}^{\infty} d t t^{s-1} \operatorname{Tr} e^{-t \frac{L_{4}}{\mu^{2}}}=\mu^{2 s} \zeta\left(s \mid L_{4}\right),
$$

valid for $\operatorname{Re} s>2$. In order to get the meromorphic structure of the function (2.12), we splits the integration range in the two intervals $[0,1)$ and $[1, \infty)$ obtaining in this way two integrals. The last one is regular for $s \rightarrow 0$, while the behaviour of the first one can be estimated by using the asymptotics, Eq. 2.11). We have

$$
\zeta\left(s \mid L_{4}\right) \sim \frac{\ell}{\sqrt{4 \pi} \Gamma(s)} \sum_{r=0}^{\infty}\left[\frac{A_{r}}{s+r-2}-\frac{P_{r}}{(s+r-2)^{2}}\right]
$$

and so the meromorphic structure reads

$$
\begin{aligned}
\zeta\left(s \mid L_{4}\right)=\frac{\ell}{\sqrt{4 \pi}}\left[\frac{A_{0}}{s-2}\right. & \left.+\frac{A_{1}}{s-1}-\frac{P_{1}}{(s-1)^{2}}-\frac{P_{2}}{s}\right] \\
& -\frac{\ell}{\sqrt{4 \pi}} \sum_{n=1}^{\infty} \frac{(-1)^{n} n ! P_{n+2}}{s+n}+\frac{\ell A_{2}}{\sqrt{4 \pi}}+J_{4}(s),
\end{aligned}
$$

where $J_{4}(s)$ is an analytic function. We see that in contrast with the usual cases, the analytic continuation of $\zeta$-function is not regular at $s=0$, but it has a simple pole. It has to be noted also the presence of a double pole at $s=1$ and new simple poles at $s=-n(n \geq 2)$. 


\section{The one-loop effective action}

We have seen in the previous Section that $\zeta$-function regularisation cannot be used without modifications. In order to understand how to proceed, we shall work within the so called propertime regularisation of one-loop effective action. More specifically, we define the regularised determinant by means of [25, ,7]

$$
\left(\ln \operatorname{det} L_{4}\right)_{\rho_{\varepsilon}}=-\int_{0}^{\infty} d t t^{-1} \rho_{\varepsilon}(t) \operatorname{Tr} e^{-t L_{4}},
$$

in which $\rho_{\varepsilon}(t)$ is a regularisation function necessary to deal with the singularities of $\operatorname{Tr} e^{-t L_{4}}$ present for $t \rightarrow 0$. The properties of $\rho_{\varepsilon}(t)$ are the following [25, 26]:

$$
\lim _{\varepsilon \rightarrow 0} \rho_{\varepsilon}(t)=1, \quad \lim _{t \rightarrow 0} \rho_{\varepsilon}(t) \sim t^{2+\delta}, \quad \delta>0 .
$$

Of course, the latter requirement is valid for a sufficiently large $\varepsilon$. The $\zeta$-function regularisation may be obtained with the choice $\rho_{\varepsilon}^{H}(t)=\frac{d}{d \varepsilon}\left(\frac{t^{\varepsilon}}{\Gamma(\varepsilon)}\right)$. In fact, for a suitable $\operatorname{Re} \varepsilon>0$ we have

$$
\left(\ln \operatorname{det} L_{4}\right)_{H}=-\int_{0}^{\infty} d t t^{-1} \frac{d}{d \varepsilon}\left(\frac{t^{\varepsilon}}{\Gamma(\varepsilon)}\right) \operatorname{Tr} e^{-t L_{4}}=-\frac{d}{d \varepsilon} \zeta\left(\varepsilon \mid L_{4}\right) .
$$

In the usual cases, namely when $\zeta\left(s \mid L_{4}\right)$ is regular at $s=0$, the $\zeta$-function regularisation directly gives the finite part of the effective potential. On the contrary, the other regularisations, besides a finite part, give divergent terms proportional to the spectral coefficients (ultraviolet divergences). For example, the cutoff proper-time regularisation, corresponding to the choice $\rho_{\varepsilon}^{C}(t)=\theta(t-\varepsilon)$, gives three ultraviolet divergent terms, proportional to $\varepsilon^{-2}, \varepsilon^{-1}, \ln \varepsilon$ respectively, while the choice $\rho_{\varepsilon}^{D}(t)=t^{\varepsilon}$ [24] (related to dimensional regularisation) gives only a divergent term $-\varepsilon^{-1} \zeta\left(0 \mid L_{4}\right)$.

As shown in the previous section, Eq. (2.14), the $\zeta$-function for our case is not regular at the point $s=0$. This means that $\zeta$-function regularisation for the effective potential gives divergent contributions as well as all other regularisations. From Eqs. (2.5), (2.7) and

$$
\begin{aligned}
\zeta\left(s \mid \frac{L_{4}}{\mu^{2}}\right)=\frac{\ell}{\sqrt{4 \pi}}\left[\frac{\tilde{A}_{0} \delta^{4}}{(s-1)(s-2)}+\frac{\tilde{A}_{1} \delta^{2}}{s-1}+\tilde{A}_{2}\right]\left(\frac{\delta^{2}}{\mu^{2}}\right)^{-s} \\
+\frac{\ell \tilde{P}_{1} \delta^{2}}{\sqrt{4 \pi(s-1)}}\left[\psi(s-1)-\ln \frac{\delta^{2}}{|\kappa|}\right]\left(\frac{\delta^{2}}{\mu^{2}}\right)^{-s}+\frac{G(s)}{\Gamma(s)},
\end{aligned}
$$

where $\psi$ is the logarithmic derivative of $\Gamma$ and $G(s)$ is a function, regular in a neighbourhood of the point $s=0$, which may be expressed in terms of the function $f(z)$, introduced in the Appendix B and defined by Eq. (B.16) and the Selberg zeta-function $Z(s)$ related to the hyperbolic contribution (see for example [7]). In particular, its value at the point $s=0$ is given by

$$
\begin{aligned}
G(0)= & \frac{\ell \delta^{2}}{\pi} \int_{1}^{\infty}\left(|\kappa| r^{2}-1\right)^{1 / 2} \frac{Z^{\prime}}{Z}(\delta r+1) d r \\
& +\frac{\ell}{2 \pi} \int_{0}^{\infty}\left(r^{2}+\delta^{2}\right)^{-\frac{1}{2}}\left[\operatorname{irf}\left(\frac{i r}{2}\right)-\frac{\sqrt{|\kappa|}}{6}\right] d r+\text { c.c. }
\end{aligned}
$$

and its computation may require a numerical calculation. As a result

$$
\begin{array}{r}
\left(\ln \operatorname{det} \frac{L_{4}}{\mu^{2}}\right)_{H}=-\frac{\ell P_{2}}{\sqrt{4 \pi} \varepsilon^{2}}+\frac{\ell P_{2}}{\sqrt{4 \pi}} \\
{\left[\frac{1}{2}\left(\ln \frac{\delta^{2}}{\mu^{2}}\right)^{2}+\left(\gamma-1+\ln \frac{\mu^{2}}{|\kappa|}\right)\left(\ln \frac{\delta^{2}}{\mu^{2}}-1\right)+\frac{\pi^{2}}{6}\right]} \\
+\frac{\ell}{\sqrt{4 \pi}}\left[A_{2} \ln \frac{\delta^{2}}{\mu^{2}}+\frac{1}{4} A_{0} \delta^{4}+A_{1} \delta^{2}\right]-G(0)+O(\varepsilon) .
\end{array}
$$


The structure of the ultraviolet divergences may be put in evidence by using, for example, the cutoff proper-time regularisation. A straightforward computation gives

$$
\begin{array}{r}
\left(\ln \operatorname{det} \frac{L_{4}}{\mu^{2}}\right)_{C}=\left(\ln \operatorname{det} \frac{L_{4}}{\mu^{2}}\right)_{H}+\gamma \frac{\ell \tilde{A}_{2}}{\sqrt{4 \pi}}-\frac{\ell P_{2}}{\sqrt{4 \pi}}\left[\gamma \ln \frac{\mu^{2}}{|\kappa|}+\frac{\gamma^{2}}{2}-\frac{\pi^{2}}{12}\right] \\
+\frac{\ell P_{2}}{\sqrt{4 \pi}}\left[\frac{1}{\varepsilon^{2}}+\frac{\mu^{2}}{\delta^{2} \varepsilon}\left(\ln \varepsilon+1-\ln \frac{\mu^{2}}{|\kappa|}\right)+\frac{1}{2}(\ln \varepsilon)^{2}+\ln \varepsilon \ln \frac{\mu^{2}}{|\kappa|}\right] \\
-\frac{\ell}{\sqrt{4 \pi}}\left[\frac{A_{0} \mu^{4}}{2 \varepsilon^{2}}+\frac{A_{1} \mu^{2}}{\varepsilon}-A_{2} \ln \varepsilon\right]
\end{array}
$$

which, apart divergent and finite terms, is equal to Eq. (3.6).

We conclude this section with some remarks. First one can see that in general the $\zeta$-function regularisation is on the same level of the other proper-time regularisations and needs a divergent counterterm. If one makes use of another regularisation new additional divergences appear. Such new ultraviolet divergences depend on the coefficient

$$
P_{2}=-\frac{\sqrt{|\kappa|}}{8 \sqrt{\pi}}\left[m^{2}+\frac{\lambda \phi_{c}^{2}}{2}+|R|\left(\frac{1}{6}-\xi\right)\right] .
$$

However, their dependence on $\phi_{c}$ is such that the usual one-loop renormalisation procedure may be applied. Furthermore, in the case of massless free scalar field with the conformal coupling $\xi=1 / 6$, we have $P_{2}=0$ and thus, in this particular case the usual evaluation of the effective action by means of $\zeta$-function methods works without any modification.

With regard to the general case, we will not discuss here the renormalization, but we limit ourselves to the following considerations. Since there exist a general proof that scale dependence of the effective potential, as well as the renormalization group equations, are standard in an arbitry curved space-time [27], one should expect that the additional divergence does not modify the standard renormalization procedure.

\section{Finite temperature effects}

Since we have an ultrastatic space-time, the finite temperature effects can be studied considering the scalar field on $S^{1} \times H^{3} / \Gamma$ satisfying periodic boundary conditions on the imaginary time, the period $\beta$ being interpreted as inverse equilibrium temperature. Making use of the $\zeta$-regularisation, the logarithmic of the partition function is

$$
\ln Z_{\beta}(\varepsilon)=-\frac{1}{2} \frac{d}{d \varepsilon}\left\{\frac{1}{\Gamma(\varepsilon)} \int_{0}^{\infty} d t t^{\varepsilon-1} \operatorname{Tr} e^{-t L_{\beta} / \mu^{2}}\right\},
$$

where by $L_{\beta}$ we indicate the Laplace-like operator acting on periodic scalar fields in $S^{1} \times H^{3} / \Gamma$, while $L_{4}$ acts on fields in $\mathbb{R} \times H^{3} / \Gamma$. One easily has

$$
\operatorname{Tr} e^{-t L_{\beta}}=\frac{\beta}{\sqrt{4 \pi t}}\left(1+2 \sum_{n=1}^{\infty} e^{-\frac{n^{2} \beta^{2}}{4 t}}\right) \operatorname{Tr} e^{-t L_{3}}=\left(1+2 \sum_{n=1}^{\infty} e^{-\frac{n^{2} \beta^{2}}{4 t}}\right) \operatorname{Tr} e^{-t L_{4}} .
$$

Here we have put

$$
\operatorname{Tr} e^{-t L_{4}}=\frac{\beta}{\sqrt{4 \pi t}} \operatorname{Tr} e^{-t L_{3}}
$$

as in the zero temperature case with $\ell \rightarrow \beta$. As a result we have

$$
\ln Z_{\beta}(\varepsilon)=-\frac{1}{2} \zeta^{\prime}\left(\varepsilon \mid \frac{L_{4}}{\mu^{2}}\right)-\int_{0}^{\infty} d t t^{-1} \sum_{n=1}^{\infty} e^{-\frac{n^{2} \beta^{2}}{4 t}} \operatorname{Tr} e^{-t L_{4}}+O(\varepsilon),
$$


in which the first contribution, depending linearly on $\beta$, is related to the vacuum energy (effective potential) and the second one is the statistical sum contribution. It is possible to show that this last contribution is ultraviolet finite, thus we may take the limit $\varepsilon \rightarrow 0$.

By using the Mellin-Parseval identity, the free energy can be conveniently written in the form [0]

$$
F_{\beta}(\varepsilon)=\frac{1}{2 \beta} \zeta^{\prime}\left(\varepsilon \mid \frac{L_{4}}{\mu^{2}}\right)+\frac{1}{2 \pi i \beta} \int_{\operatorname{Re} z=c} \Gamma(z) \zeta\left(z \mid L_{4}\right)\left(\frac{\beta}{2}\right)^{-2 z} \Gamma(z) \zeta_{R}(2 z) d z,
$$

where $\zeta_{R}(z)$ is the Riemann $\zeta$-function and $c>2$. The integrand function has double poles at the points $z=-1,-2,-3, \ldots$ and $z=1$, simple poles at $z=\frac{1}{2}$ and $z=2$ and finally a pole of order 3 at $z=0$. Shifting the vertical contour to the left, with the help of the theorem of residues we obtain a small $\beta$ expansion for the statistical sum $\hat{F}_{\beta}=F_{\beta}(\varepsilon)-F_{\infty}(\varepsilon)$. It reads

$$
\begin{aligned}
\hat{F}_{\beta}=\frac{V(F) \pi^{2}}{90 \beta^{4}} & +\frac{\pi^{\frac{3}{2}}}{3 \beta^{2}}\left[A_{1}+2 P_{1}\left(\gamma+\ln \frac{\beta}{2}-\frac{6 \zeta_{R}^{\prime}(2)}{\pi^{2}}\right)\right]+\frac{\zeta^{\prime}\left(0 \mid L_{3}\right)}{2 \beta} \\
& +\frac{P_{2}}{\sqrt{4 \pi}}\left[A_{2}+2 P_{2}\left(\gamma+2 \zeta_{R}^{\prime}(0)+\frac{1}{2} \ln \beta\right)\right] \ln \beta+\ldots
\end{aligned}
$$

It should be noted that the presence of parabolic elements in the discrete group of isometry leads to the appearance of new terms in the high temperature expansion, only the leading (Planckian) one being left unchanged.

\section{Conclusions}

In this paper we have considered a self-interacting scalar field in an ultrastatic space-time whose spatial section is a non-compact hyperbolic manifold with finite volume. By means of the Selberg trace formula we have investigated the one-loop effective action. It has been shown that the asymptotics of the heat-kernel trace related to the small disturbance operator, a Laplacetype operator acting on the manifold $R \times H^{3} / \Gamma$, contains logarithmic terms in $t$, absent in the smooth or compact background. As a consequence, we have seen that the $\zeta$-function can be analytically continued and its meromorphic structure admits a simple pole at $s=0$. In order to analyse this new situation, a general class of regularisations (proper-time regularisations) have been considered and we have observed that also the $\zeta$-function regularisation of the one-loop effective action requires a divergent counterterm, as well as all other regularisations. We have noticed, however, that no problem arises as soon as one is dealing with a conformally coupled free massless scalar field.

The finite temperature effects have also been investigated and a general expression for the one-loop free energy at temperature $\beta^{-1}$ has been presented. It has to be noticed that new terms appear in the high-temperature expansion. As a consequence, the quantum corrections to the mass, which are defined by means of the relation

$$
V\left(\phi_{c}\right)=\Lambda_{e f f}+\frac{1}{2}\left[m^{2}+m^{2}(\beta)\right] \phi_{c}^{2}+O\left(\phi_{c}^{4}\right),
$$

receive new contributions, which may play a role in the discussion of the phase transition of the system, that is in the problem of symmetry breaking (or restoration) in some cosmological scenarios.

Another potentially interesting application of the model we have studied deals with the problem of the divergences one encounters in the evaluation of the entropy (the entanglement entropy) for a scalar field in Rindler space-time and in the related mechanical statistical entropy of the black hole [28, 29, 30]. This issue has been recently discussed in Ref. [31]. It is a common feature of the all methods used in the analysis of the mechanical-statistical entropy, namely the 
entropy of quantum fields in a background with a horizon, the fact that the density of states is blowing up near the horizon. It has been shown that the leading divergence is associated to the divergence of the spatial optical volume. For Rindler space-time, the 4-dimensional conformal optical metric is ultrastatic and the spatial optical section turns out to be locally the hyperbolic 3-dimensional space $H^{3}$, which obviously, being non-compact, has an infinite volume. For physical reasons, the spatial metric must be non-compact. However, we remind that, besides the huge number of compact hyperbolic manifolds (the ones whose discrete group of isometries contain hyperbolic and elliptic elements), there exist also non-compact hyperbolic manifolds with finite volume.

With regard to this issue, it might be interesting to consider the space-time we are dealing with, i.e. $R \times H^{3} / \Gamma$ as the conformal optical counterpart of a space-time which (at least locally) is the Rindler one with some identifications. As has been considered in this paper, the discrete group $\Gamma$ has the form $S L(2, \mathbb{Z}+i \mathbb{Z}) /\{ \pm I d\}$, generated by parabolic mappings and related to non-compact manifold with invariant fundamental domain of finite volume. In the Appendix A a homeomorphism between $H^{3} / \Gamma$ and boundaryless manifold $\left(S^{1} \times U_{c}\right) / \partial M$ has been presented. The manifold $\left(S^{1} \times U_{c}\right) / \partial M$ can be constructed by means of the compactification of two planar coordinates of a Rindler-like spatial section and the identification of the faces of the corresponding polyhedron. In some sense, this compactification procedure could be similar to the regularisation with the help of volume cutoff parameter introduced in Ref. [31].

\section{Acknowledgments}

We thank K. Kirsten and L. Vanzo for useful discussions. A.A.B. would like to thank I.N.F.N. for financial support and Prof. M. Toller for kind hospitality at Department of Physics, University of Trento. The research of A.A.B. was supported in part by Russian Foundation for Fundamental Research grant No. 95-02-03568-a and by Russian Universities grant No. 95-0-6.4-1.

\section{A Fundamental domain of the discrete group $S L(2, \mathbb{Z}+i \mathbb{Z}) /\{ \pm I d\}$}

In this first Appendix, we will summarise the geometry and local isometry associated with a simple 3-dimensional complex Lie group. We shall consider discrete subgroups $\Gamma \subset S L(2, \mathbb{C}) /\{ \pm I d\}$, where $I d$ is the $2 \times 2$ identity matrix and is an isolated element of the $\Gamma$. The group $\Gamma$ acts discontinuously at the point $z \in \overline{\mathbf{C}}, \overline{\mathbb{C}}$ being the extended complex plane. We recall that a transformation $\gamma \neq I d \gamma \in \Gamma$, with

$$
\gamma z=\frac{a z+b}{c z+d}, \quad a d-b c=1, \quad a, b, c, d \in \mathbb{C},
$$

is called elliptic if $(\operatorname{Tr} \gamma)^{2}=(a+d)^{2}$ satisfies $0 \leq(\operatorname{Tr} \gamma)^{2}<4$, hyperbolic if $0 \leq(\operatorname{Tr} \gamma)^{2}>$ 4, parabolic if $(\operatorname{Tr} \gamma)^{2}=4$ and loxodromic if $(\operatorname{Tr} \gamma)^{2} \in \mathbb{C} \backslash[0,4]$. The classification of these transformations can also be based on the properties of their fixed points, the number of which is one for the parabolic transformations and 2 for the other cases.

The element $\gamma \in S L\left(2, \mathbb{C}\right.$ ) acts on $z=(y, w) \in H^{3}$ (as usual here we choose units in which $\kappa=-1), w=x_{1}+i x_{2}$ by means of the following linear-fractional transformation:

$$
\gamma z=\left(\frac{y}{|c w+d|^{2}+|c|^{2} y^{2}}, \frac{(a w+b)(\bar{c} \bar{w}+\bar{d})+a \bar{c} y^{2}}{|c w+d|^{2}+|c|^{2} y^{2}}\right) .
$$

ffl

The isometric circle of a transformation $\gamma \in S L(2, \mathbb{C}) /\{ \pm I d\}$ for which $\infty$ is not a fixed point is defined to be the circle

$$
I(\gamma)=\{z: \quad|\gamma z|=1\}, \quad \text { or } \quad I(\gamma)=\left\{z: \quad|z+d / c|=|c|^{-1}\right\}, \quad c \neq 0 .
$$


A transformation $\gamma$ carries its isometric circle $I(\gamma)$ into $I\left(\gamma^{-1}\right)$.

The isometric fundamental domain of a Fuchsian group (Kleinian group without loxodromic elements) has the following structure: it is bounded by arcs of circles orthogonal to the invariant circle and consists either of two symmetric components or of a single component, while the mappings connecting its equivalent sides, generate the whole group. In many cases, it is more convenient to deal with other fundamental regions. For example, the so-called normal fundamental Dirichlet polygons are often used for Fuchsian groups and we will follow this approach here.

Now we consider a discrete group $\Gamma$ of a special kind. Let $G=S L(2, \mathbb{C}) /\{ \pm I d\}$, then for $\Gamma \subset G$, one can choose $\Gamma=S L(2, \mathbb{Z}+i \mathbb{Z}) /\{ \pm I d\}$, where $\mathbb{Z}$ is the ring of integer numbers. The element $\gamma \in \Gamma$ will be identified with $-\gamma$. The group $\Gamma$ has, within a conjugation, one maximal parabolic subgroup $\Gamma_{\infty}(c=0)$. Thus, the fundamental domain related to $\Gamma$ has one parabolic vertex and can be taken in the form 33, 33]

$$
F(\Gamma)=\left\{(y, w): x_{1}^{2}+x_{2}^{2}+y^{2}>1, \quad-\frac{1}{2}<x_{2}<x_{1}<\frac{1}{2}\right\} .
$$

As an example let us consider the parabolic mappings

$$
g_{1}(z)=z+1, \quad g_{2}(z)=z+i .
$$

If we identify the faces of the polyhedron, Eq. (A.4), we get a manifold $M(\Gamma)$ that is homeomorphic to a punctured torus $S^{1} \otimes S^{1} \otimes\left[-\frac{1}{2}, \frac{1}{2}\right)=U_{c} \otimes S^{1}$, where $U_{c}=\left\{z: \quad 0<|z| \leq \frac{1}{2}\right\}$ is a punctured cylinder. It is turned into a hyperbolic manifold by removing the boundary $\partial M(\Gamma)$, which is homeomorphic to the torus $S^{1} \otimes S^{1}$.

\section{B A contribution to the Selberg trace formula associated with the cusp form}

Here we will discuss the Selberg trace formula on the space $H^{3}$, which can be constructed as an expansion in eigenfunctions of the automorphic Laplacian. To begin with, we assume that the group $\Gamma$ is generated by parabolic mappings. Since the discrete group $\Gamma$ has a cusp at $\infty$ $(c=0)$, each element of the stabiliser $\Gamma_{\infty}$ is a translation, as we have seen in the Appendix A. We compute the conjugacy class $\{\gamma\}_{\Gamma}, \gamma \in \Gamma_{\infty}$ with $\gamma$ different from identity. If

$$
\gamma=\left(\begin{array}{cc}
1 & n_{1}+i n_{2} \\
0 & 1
\end{array}\right), \quad n_{1}, n_{2} \in \mathbb{Z},
$$

then the conjugacy class with representative $\gamma$ consists in $\gamma$ and $\gamma^{-1}$, where

$$
\gamma^{-1}=\left(\begin{array}{cc}
1 & -n_{1}-i n_{2} \\
0 & 1
\end{array}\right)
$$

The remaining conjugacy classes have the representatives in $\Gamma_{\infty}$ of the form

$$
\gamma_{1}=\left(\begin{array}{cc}
i & 0 \\
0 & -i
\end{array}\right), \gamma_{2}=\left(\begin{array}{cc}
i & 1 \\
0 & -i
\end{array}\right), \gamma_{3}=\left(\begin{array}{cc}
i & -i \\
0 & -i
\end{array}\right), \gamma_{4}=\left(\begin{array}{cc}
i & 1-i \\
0 & -i
\end{array}\right)
$$

The centralisers related to these representations read

$$
\begin{aligned}
& \Gamma^{\gamma}=\left(\begin{array}{cc}
1 & m_{1}+i m_{2} \\
0 & 1
\end{array}\right), \quad m_{1}, m_{2} \in \mathbb{Z}, \\
& \Gamma^{1}=\Gamma^{\gamma_{1}}=\left\{\left(\begin{array}{ll}
1 & 0 \\
0 & 1
\end{array}\right),\left(\begin{array}{cc}
i & 1 \\
0 & -i
\end{array}\right),\left(\begin{array}{cc}
0 & 1 \\
-1 & 0
\end{array}\right),\left(\begin{array}{ll}
0 & i \\
i & 0
\end{array}\right)\right\},
\end{aligned}
$$




$$
\begin{aligned}
& \Gamma^{2}=\Gamma^{\gamma_{2}}=\left\{\left(\begin{array}{ll}
1 & 0 \\
0 & 1
\end{array}\right),\left(\begin{array}{cc}
i & 1 \\
0 & -i
\end{array}\right),\left(\begin{array}{cc}
i & 0 \\
2 & -i
\end{array}\right),\left(\begin{array}{cc}
-1 & i \\
2 i & 1
\end{array}\right)\right\}, \\
& \Gamma^{3}=\Gamma^{\gamma_{3}}=\left\{\left(\begin{array}{ll}
1 & 0 \\
0 & 1
\end{array}\right),\left(\begin{array}{cc}
i & -i \\
0 & -i
\end{array}\right),\left(\begin{array}{cc}
1 & -1 \\
2 & -1
\end{array}\right),\left(\begin{array}{cc}
i & 0 \\
2 i & -i
\end{array}\right)\right\}, \\
& \Gamma^{4}=\Gamma^{\gamma_{4}}=\left\{\left(\begin{array}{ll}
1 & 0 \\
0 & 1
\end{array}\right),\left(\begin{array}{cc}
i & 1-i \\
0 & -i
\end{array}\right),\left(\begin{array}{cc}
i & 0 \\
1+i & -i
\end{array}\right),\left(\begin{array}{cc}
1 & -1-i \\
1-i & -1
\end{array}\right)\right\} .
\end{aligned}
$$

Let us consider an arbitrary integral operator with kernel $k\left(z, z^{\prime}\right)$. Invariance of the operator is equivalent to fulfillment of the condition $k\left(\gamma z, \gamma z^{\prime}\right)=k\left(z, z^{\prime}\right)$ for any $z, z^{\prime} \in H^{3}$ and $\gamma \in$ $P S L(2, \mathbb{C})$. So the kernel of the invariant operator is a function of the geodesic distance between $z$ and $z^{\prime}$. It is convenient to replace such a distance with the fundamental invariant of a pair of points $u\left(z, z^{\prime}\right)=\left|z-z^{\prime}\right|^{2} / y y^{\prime}$, thus $k\left(z, z^{\prime}\right)=k\left(u\left(z, z^{\prime}\right)\right)$. Let $\lambda_{i}$ be the isolated eigenvalues of the self-adjoint extension of the Laplace operator and let us introduce a suitable analytic function $h(r)$ and $r_{j}^{2}=\lambda_{j}-1$. It can be shown that $h(r)$ is related to the quantity $k(u(z, \gamma z))$ by means of the Selberg transform. Let us denote by $g(u)$ the Fourier transform of $h(r)$, namely

$$
g(u)=\frac{1}{2 \pi} \int_{-\infty}^{\infty} e^{-i r u} h(r) d r
$$

For one parabolic vertex let us introduce a subdomain $F_{Y}$ of the fundamental region $F(\Gamma)$ by means

$$
F_{Y}=\{z \in F(\Gamma), z=\{y, \mathbf{x}\} \mid y \leq Y\},
$$

where $Y$ is a sufficiently large positive number. Let us suppose that $h(r)$ is a even analytic function in the strip $|\operatorname{Im} r|<1+\varepsilon(\varepsilon>0)$ and $h(r)=O\left(1+|r|^{2}\right)^{-2}$. Then for $N=3$ the following formula holds [32]:

$$
\begin{aligned}
\sum_{j} h\left(r_{j}\right)=\lim _{Y \rightarrow \infty}\left\{\int_{F_{Y}} \sum_{\gamma \in \Gamma} k(u(z, \gamma z)) d \mu(z)\right. \\
\left.\quad-\frac{1}{2 \pi} \int_{0}^{\infty} h(r) \int_{F_{Y}}|E(z, 1+i r)|^{2} d \mu(z) d r\right\},
\end{aligned}
$$

in which $d \mu(z)=y^{-3} d y d x_{1} d x_{2}$ is the invariant measure on $H^{3}$ and $E(z, s)$ is the EisensteinMaass series associated with one cusp, namely

$$
E(z, s)=\sum_{\gamma \in\left(\Gamma / \Gamma_{\infty}\right)} y^{s}(\gamma z), \quad x_{2}(z)=\operatorname{Im} z
$$

The series (B.8) converges absolutely for Re $s>1$ and uniformly in $z$ on compact subset of $H^{3}$. All poles of $E(z, s)$ are contained in the union of the region $\operatorname{Re} s<1 / 2$ and the interval $[1 / 2,1]$ and those contained in such an interval are simple. Furthermore, for each $s$, the series $E(z, s)$ is a real analytic function on $H^{3}$, automorphic relative to the group $\Gamma$ and satisfies the eigenvalues equation

$$
\bar{\Delta}_{3} E(z, s)=s(s-1) E(z, s) .
$$

The asymptotic expansion of the second integral in Eq. (B.7) can be found with the help of Maass-Selberg relation (for details see Ref. [32]). For $Y \rightarrow \infty$ one has the following result

$$
\begin{aligned}
\frac{1}{2 \pi} \int_{0}^{\infty} h(r) \int_{F_{Y}}|E(z, 1+i r)|^{2} d \mu(z) d r= & g(0) \ln Y+\frac{h(0)}{4} S(1) \\
& -\frac{1}{4 \pi} \int_{-\infty}^{\infty} h(r) \frac{S^{\prime}(1+i r)}{S(1+i r)} d r+O(1) .
\end{aligned}
$$


The function $S(s)$ (in the general case it is the S-matrix) is given by a generalised Dirichlet series, convergent for $\operatorname{Re} s>1$,

$$
S(s)=\frac{\sqrt{\pi} \Gamma\left(s-\frac{1}{2}\right)}{\Gamma(s)} \sum_{c \neq 0} \sum_{0 \leq d<|c|}|c|^{-2 s},
$$

in which the sums are taken over all pairs $c, d$ of the matrix $\left(\begin{array}{ll}* & * \\ c & d\end{array}\right) \subset \Gamma_{\infty} \backslash \Gamma / \Gamma_{\infty}$. Also the poles of the meromorphic function $S(s)$ are contained in the region $\operatorname{Re} s<1 / 2$ and in the interval $[1 / 2,1]$. The functions $E(z, s)$ and $S(s)$ can be analytically extented on the whole complex s-plane, where they satisfy the functional equations

$$
S(s) S(1-s)=I d, \quad \overline{S(s)}=S(\bar{s}), \quad E(z, s)=S(s) E(z, 1-s) .
$$

It should be noted that the terms of the trace formula associated with the elements $\gamma$ and $\gamma^{-1}$ coincide. Then the contribution to the first integral of Eq. (B.7), which comes from all conjugacy classes of the $\gamma$-type $\left(\gamma \in \Gamma^{\gamma}\right)$, for $Y \rightarrow \infty$ can be written as follows

$$
\begin{aligned}
\int_{F_{Y}} \sum_{\gamma \in \Gamma} k(u(z, \gamma z)) d \mu(z)= & (\ln Y+C) g(0)+\frac{h(0)}{4} \\
& -\frac{1}{4 \pi} \int_{-\infty}^{\infty} h(r) \psi\left(1+\frac{i r}{2}\right) d r+O(1),
\end{aligned}
$$

where $\psi$ is the logarithmic derivative of the Euler $\Gamma$-function and $C$ a computable constant which reads 32

$$
\begin{aligned}
C & =\frac{5 \ln 2}{16}-\frac{\gamma}{2}+C_{0}, \\
C_{0} & =\lim _{N \rightarrow \infty} \frac{1}{4 \pi} \sum_{i=1}^{N}\left[\left|\xi^{i}\right|^{-2}-2 \pi \ln \frac{\left|\xi^{i+1}\right|}{\left|\xi^{i}\right|}\right]-\frac{1}{2} \ln \left|\xi^{1}\right| .
\end{aligned}
$$

In the latter equation $\gamma$ is the Euler-Mascheroni constant and $\xi$ is a two-dimensional vector, such that $\gamma z=\{y, \omega+\xi\}, \xi \neq 0,\left|\xi^{i+1}\right| \geq\left|\xi^{i}\right|$.

For the derivation of the Selberg trace formula, which we have used in Sec. 3, one has to consider the contributions coming from the identity element in $\Gamma$, the normalised EisensteinMaass series, Eq. (B.8) and all $\gamma$-type conjugacy classes, Eq. (B.13). The final result reads

$$
\begin{aligned}
\sum_{j} h\left(r_{j}\right)- & \frac{1}{4 \pi} \int_{-\infty}^{\infty} h(r) \frac{S^{\prime}(1+i r)}{S(1+i r)} d r+\frac{h(0)}{4} S(1) \\
= & V(F) \int_{0}^{\infty} \frac{r^{2}}{2 \pi^{2}} h(r) d r+\sum_{\gamma} \sum_{k=1}^{\infty} \frac{\chi(\gamma)}{S_{3}(\gamma, k)} g\left(k l_{\gamma}\right) \\
& +C g(0)+\frac{h(0)}{4}-\frac{1}{4 \pi} \int_{-\infty}^{\infty} h(r) \psi\left(1+\frac{i r}{2}\right) d r \\
= & V(F) \int_{0}^{\infty} \frac{r^{2}}{2 \pi^{2}} h(r) d r+\sum_{\gamma} \sum_{k=1}^{\infty} \frac{\chi(\gamma)}{S_{3}(\gamma, k)} g\left(k l_{\gamma}\right)+\left(C+\frac{1}{2} \ln 2\right) g(0) \\
& -\frac{1}{2 \pi} \int_{0}^{\infty} h(r) \ln r d r+\frac{1}{2 \pi i} \int_{-\infty}^{\infty} h^{\prime}(r) f\left(\frac{i r}{2}\right) d r
\end{aligned}
$$

The first term in the r.h.s. of Eq. (B.15) is the contribution of the identity element, $V(F)$ is the (finite) volume of the fundamental domain $F$ with respect to the measure $d \mu$ and the second term is the standard contribution of hyperbolic elements, which has been considered, for 
example, in Ref. [7] and we refer to this reference for details. The function $f(z)$ is defined by (see Ref. [34)

$$
f(z)=\frac{1}{2} \sum_{k=1}^{\infty} \frac{k}{(k+1)(k+2)} \sum_{n=1}^{\infty} \frac{1}{(n+z)^{k+1}}
$$

and has an aymptotic expansion for large $|z|$ in terms of the Bernoulli numbers $B_{k}$. It reads

$$
f(z) \sim \sum_{k=1}^{\infty} \frac{B_{2 k}}{2 k(2 k-1) z^{2 k-1}} .
$$

\section{References}

[1] N.D. Birrell and P.C.W. Davies. Quantum Fields in Curved Space. Cambridge University Press, Cambridge, (1982).

[2] S.A. Fulling. Aspects of Quantum Field Theory in Curved Space-Time. Cambridge University Press, Cambridge, (1989).

[3] R. Camporesi. Phys. Rep. 196, 1 (1990).

[4] E. Elizalde, S. D. Odintsov, A. Romeo, A.A. Bytsenko and S. Zerbini. Zeta Regularization Techniques with Applications. World Scientific, Singapore, (1994).

[5] G. Kennedy. Phys. Rev. D 23, 2884 (1981).

[6] P. Chang and J.S. Dowker. Nucl. Phys. B395, 407 (1993).

[7] A.A. Bytsenko, G. Cognola, L. Vanzo and S. Zerbini. Phys. Rep. 266, 1 (1996).

[8] A.B. Venkov. Spectral theory of authomorphic functions and its applications. Kluwer Academic Publishers, Dordrecht, The Netherlands, (1990). Mathematics and Its Applications (Soviet Series) vol. 51.

[9] J. Elstrod, F. Grunewald and J. Mennicke. Banach Center Publ. 17, 83 (1985).

[10] J. Elstrodt, F. Grunewald and J. Mennicke. Math. Ann. 277, 655 (1987).

[11] A.A. Bytsenko, G. Cognola and L. Vanzo. J. Math. Phys. 33, 3108 (1992), Errata: J. Math. Phys., 34, 1614 (1993).

[12] G.F.R. Ellis. Gen. Relativ. Gravit. 2, 7 (1971).

[13] A. Ishibashi, T. Koike, M. Siino and S. Kojima, Compact Hyperbolic Universe and Singularities. TIT/HEP-330/COSMO-72 gr-qc/9605041 (1996).

[14] I. Efrat. Commun. Math. Phys. 119, 443 (1988).

[15] W. Müller. Invent. Math. 109, 265 (1992).

[16] D.B. Ray and I.M. Singer. Advances in Math. 7, 145 (1971).

[17] S.W. Hawking. Commun. Math. Phys. 55, 133 (1977).

[18] M. Bordag, K. Kirsten and J.S. Dowker, Heat-kernels and Functionals Determinants on the Generalized Cone. Univ. Leipzig and Univ. Manchester hep-th/9602089 (1996), to appear in Commun. Math. Phys. 
[19] J. Cheeger. J. Diff. Geom. 18, 575 (1983).

[20] C. Callias. Commun. Math. Phys. 88, 357 (1983).

[21] J. Bruning and R. Seeley. Adv. in Maths. 58, 133 (1985).

[22] G. Cognola, K. Kirsten, L. Vanzo and S. Zerbini. Phys. Rev. D 49, 5307 (1994).

[23] G. Cognola, K. Kirsten and L. Vanzo. Phys. Rev. D 49, 1029 (1994).

[24] J.S. Dowker and R. Critchley. Phys. Rev. D 13, 3224 (1976).

[25] R.D. Ball. Phys. Rep. 182, 1 (1989).

[26] G. Cognola, K. Kirsten and S. Zerbini. Phys. Rev. D 48, 790 (1993).

[27] I.L. Buchbinder, S.D. Odintsov and I.L. Shapiro. Effective Action in Quantum Gravity. A. Hilger, Bristol, (1992).

[28] G.'t Hooft. Nucl. Phys. B256, 727 (1985).

[29] L. Bombelli, R. Koul, J. Lee and R. Sorkin. Phys. Rev. D 34, 373 (1986).

[30] C.G. Callan and F. Wilczek. Phys. Lett. B333, 55 (1994).

[31] V.P. Frolov, D.V. Fursaev and A.I. Zelnikov. Phys. Rev. D 54, 2711 (1996).

[32] A. B. Venkov. Proc. Steklov Math. Inst. 125, 3 (1973).

[33] S. Lang. SL(2,R). Springer-Verlag, (1985).

[34] I.S. Gradshteyn and I.M. Ryzhik. Table of Integrals, Series and Products. Academic Press, New York, (1980). 